\title{
MACC1 upregulation promotes gastric cancer tumor cell metastasis and predicts a poor prognosis
}

\author{
Qiu-ping XIE ${ }^{\S 1}$, Cheng XIANG ${ }^{\S 1}$, Gang WANG ${ }^{2}$, Ke-feng $\mathrm{LEI}^{3}$, Yong WANG ${ }^{\dagger 1}$ \\ ( ${ }^{1}$ Department of General Surgery, the Second Affiliated Hospital, School of Medicine, Zhejiang University, Hangzhou 310009, China) \\ ( ${ }^{2}$ Department of Colon Surgery, the Cancer Hospital of Zhejiang Province, Hangzhou 310022, China) \\ ( ${ }^{3}$ Department of General Surgery, Zhejiang Provincial People's Hospital, Hangzhou 310001, China) \\ †E-mail: yushengxqp@yeah.net; drchengxiang@126.com; Surgwy@163.com \\ Received Sept. 27, 2015; Revision accepted Dec. 27, 2015; Crosschecked Apr. 15, 2016
}

\begin{abstract}
In various studies, metastasis associated with colon cancer 1 (MACC1) has been frequently reported to be abnormally highly expressed in human lung cancer, colon cancer, and hepatocellular carcinoma. Our study focuses on the association of MACC1 expression with gastric cancer (GC). During our experiment, the MACC1 expression was tested in $105 \mathrm{GC}$ samples using an immunohistochemical (IHC) method. The clinical characteristics and prognosis of these patients were summarized. During analysis, MACC1 distribution in GC samples with distant metastasis was higher than that in normal samples and in tumors with no dissemination. Subsequently, a lower 5-year survival rate had a strong correlation with high MACC1 expression. As a consequence, the present results suggest that MACC1 is more frequently expressed in a poor prognosis phenotype of $\mathrm{GC}$ and acts as a promising prognostic prediction parameter for GC.
\end{abstract}

Key words: Gastric cancer, MACC1, Prognosis, Metastasis http://dx.doi.org/10.1631/jzus.B1500236

\section{Introduction}

Gastric cancer (GC) is the fourth most frequently diagnosed cancer and ranks as the third leading cause of death from cancer worldwide. In 2011, a global analysis showed that 989600 new GC cases and as many as 738000 patient deaths occurred in 2008 . Among the population, $70 \%$ of cases occur in developing countries, especially in eastern Asia (Jemal et al., 2011). Over two-thirds of cases present with advanced or inoperable disease once diagnosed, and the prognosis is poor (MacDonald, 2006). Despite the

\footnotetext{
${ }^{\ddagger}$ Corresponding author

${ }^{\S}$ The two authors contributed equally to this work

* Project supported by the National Natural Science Foundation of China (No. 30901445)

(1) ORCID: Qiu-ping XIE, http://orcid.org/0000-0002-4658-5935; Yong WANG, http://orcid.org/0000-0002-2034-6709

C Zhejiang University and Springer-Verlag Berlin Heidelberg 2016
}

wide usage of advanced surgical techniques (Lee et al., 2008) and chemotherapy (Sun et al., 2009), the overall recovery rate for patients continues to remain poor (Morabito et al., 2009). GC pathogenesis is a complex, multistage, and heritage-related process. So far, the researches associated with GC mechanisms are far from understood. Various pathological and epidemiological studies have provided evidence that genetic factors play a crucial role in gastric carcinogenesis (González et al., 2002; Hamajima et al., 2006).

As a feature of malignant tumors, metastasis is mainly related to $\mathrm{GC}$ prognosis and the major factors of GC recurrence. Furthermore, lymph node and peritoneal metastasis are highly associated with a poor $\mathrm{GC}$ prognosis. We are very interested in the undiscovered molecular changes in distant metastasis tumors. In colon cancer, metastasis associated with colon cancer $1(M A C C 1)$ gene was first identified to be differentially expressed by a gene expression contrast 
search by comparing normal colon mucosa, primarystage tumors, and metastases lesions (Stein et al., 2009). It induces tumor proliferation, metastasis, and invasion in in vitro colon epithelium cancer cell culture, as well as in colon cancer patients (Arlt and Stein 2009). Besides this, MACC1 acts as the main regulator factor of the HGF/Met pathway, and influences cell activity through downstream MAPK upregulation (Stein et al., 2009). In previous studies, high MACC1 expression was found to be present in various cancer tissues including colon cancer (Shirahata et al., 2010a), hepatocarcinoma (Shirahata et al., 2011), and lung adenocarcinoma (Chundong et al., 2011; Shimokawa et al., 2011).

Accumulating evidence suggests that the high expression of MACC1 has been proved to be associated with increased metastasis risk and poorer patient survival in various types of cancers including bladder urothelial carcinoma (Xu et al., 2015), cervical cancer (Zhou et al., 2015), and colorectal cancer (Weidle et al., 2015). Recently, upregulation of MACC1 expression was reported in GC (Shirahata et al., 2010b). MACC1 was reported to play a key role in GC metastasis (Wang et al., 2013), epithelial-mesenchyal transition (EMT) phenomena (Huang et al., 2015), and angiogenesis (Sun et al., 2015). Until now, the role of MACC1 in GC has still remained controversial (Ge et al., 2011). During our work, we investigated MACC1 expression in surgical specimens from 105 GC patients, and we identified the correlation between MACC1 expression and various clinic pathological parameters.

\section{Materials and methods}

\subsection{Patients and tissue samples}

GC tissue was obtained from 105 patients who were pathologically proven and underwent surgical operation between January 2004 and December 2006 in our hospital. None of the 105 patients had received neoadjuvant before operation. The tissue samples were formalin-fixed and paraffin-embedded following surgical removal, then were cut into thick sections (4 $\mu \mathrm{m})$ and mounted onto glass slides for further protein expression analysis. The clinical characteristics of these patients, such as gender, age, tumor location, differentiation, and tumor-node-metastasis
(TNM) stage, are summarized in Table 1. Follow-up by consultation of the case documents or by telephone was performed until death or June 2011, whichever came first. The clinical stage of the tumors was determined according to the TNM classification of the International Union Against Cancer (2009) (Edge and Compton, 2010). Thirty normal tissue specimens from gastritis patients without malignancy undergoing endoscopic biopsy were collected as blank controls. The present study was approved by the Ethical Committee of the Second Affiliated Hospital of Zhejiang University and the Zhejiang Provincial People's Hospital, China.

\subsection{Immunohistochemical staining of MACC1}

Immunohistochemical (IHC) examination of MACC1 was performed using rabbit anti-MACC1 polyclonal antibody (Abcam, Hong Kong, China) on the specimens described previously. Tissue sections were first incubated at $60{ }^{\circ} \mathrm{C}$ for $2 \mathrm{~h}$, followed by deparaffinizing them in xylene; then they were rehydrated in a list of graded alcohols. For retrieval of the MACC1 antigen, the sections were boiled in a solution of $1 \%(\mathrm{v} / \mathrm{v})$ citrate buffer for $20 \mathrm{~min}$. After natural cooling, the specimens were incubated with $3 \%$ (v/v) $\mathrm{H}_{2} \mathrm{O}_{2}$ in methanol for another 10 min to block endogenous tissue peroxidase activity, followed by rabbit anti-MACC1 incubation in a moist chamber overnight at $4{ }^{\circ} \mathrm{C}$. Negative controls were managed with normal goat serum instead of the primary antibody. Subsequently, the specimens were incubated with a horseradish peroxidase (HRP)-conjugated secondary antibody (Beijing Zhongshan Biotechnology, China) for $35 \mathrm{~min}$ at room temperature. Finally, the slides were incubated with 3,3'-diaminobenzidine (DAB) substrate (Beijing Zhongshan Biotechnology) for $7 \mathrm{~min}$, counterstained with hematoxylin, dehydrated, and then coverslipped.

\subsection{Staining evaluation}

All the immunostained sections were evaluated independently using light microscopy (Olympus, Japan) by two expert pathologists who were blinded from the clinical details. If scores differed between the two investigators, the sections were reassessed by simultaneous dual examination with a multihead microscope. A hundred cells were randomly chosen, and the MACC1-positive cells were filtered 
from five average isolated fields of each section. An immunoreactivity score (IRS) system was performed. The extensional criteria were defined as follows: (i) frequency of positive cells: $0(\leq 5 \%$ positively stained cells), 1 (6\%-25\% positively stained cells), 2 (26\%-50\% positively stained cells ), 3 (51\%-75\% positively stained cells), 4 ( $>75 \%$ positively stained cells); (ii) staining intensity: 0 (no staining), 1 (weak staining, pallide-flavens), 2 (moderate staining, yellow), 3 (strong staining, brown). When (i) and (ii) were multiplied, the final score was stratified as: $-(0$, negative $),+(1-4$, weakly positive $),++(5-8$, moderately positive), $+++(9-16$, strongly positive). In this study, - to + were used to indicate tumors with low MACC1 expression, and ++ to +++ were used to define high MACC1 expression.

\subsection{Statistical analysis}

All statistical analyses were performed with SPSS software 18.0 (SPSS Inc., Chicago, IL, USA). Correlations between MACC1 level and clinic pathological features such as patient survival rate were analyzed using the $\chi^{2}$ method. Survival curves were calculated and drawn by the Kaplan-Meier method. A log-rank test was used to analyze the differences between the two curves. $P<0.05$ was validated as the statistical significance.

\section{Results}

\subsection{Detection of MACC1 expression by the rela- tionship between IHC staining and patients' clinic pathological factors}

MACC1 was predominantly localized in the cytoplasm or membrane of GC cells. Indeed, MACC1 was expressed in $74.3 \%(78 / 105)$ of tumor samples, whereas the expression was not detected in normal mucosa $(P<0.01)$. Some $16(15.2 \%)$ out of the 105 patients had tumor distant metastasis; IHC analysis revealed a significant increase in the intensity of MACC1 of metastasis tumors $(12 / 16,75.0 \%)$ compared with normal samples and tumors with no dissemination $(38 / 89,42.7 \%)(P=0.017$; Fig. 1$)$. Meanwhile, no significant association was found between high expression of MACC1 and clinical factors (patient gender, age, size, location, histological differentiation, invasion depth, and TNM stages) (Table 1).

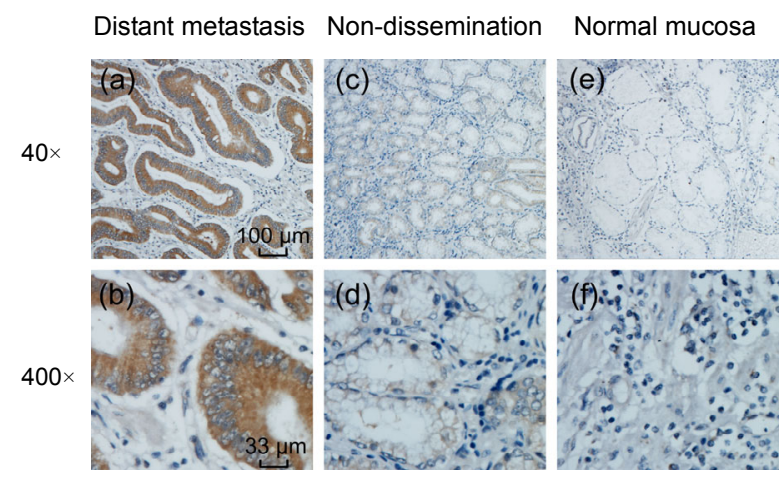

Fig. 1 IHC analysis of MACC1 in gastric cancer tissue and normal mucosa

$(\mathrm{a}, \mathrm{b})$ Distant metastasis adenocarcinoma $(+++), 40 \times$ and 400×; (c, d) Non-dissemination adenocarcinoma, (+-) 40× and $400 \times$; (e, f) Normal adjacent gastric mucosa, $(-) 40 \times$ and $400 \times$

Table 1 Relationship of MACC1 with gastric cancer pathologic parameters

\begin{tabular}{|c|c|c|c|c|c|}
\hline \multirow{2}{*}{$\begin{array}{l}\text { Clinical } \\
\text { parameter }\end{array}$} & \multirow{2}{*}{ Case } & \multicolumn{2}{|c|}{ MACC1 expression } & \multirow{2}{*}{$\chi^{2}$} & \multirow{2}{*}{$P$-value } \\
\hline & & Low & High & & \\
\hline Gender & & & & 0.022 & 0.883 \\
\hline Female & 28 & $15(53.6 \%)$ & $13(46.4 \%)$ & & \\
\hline Male & 77 & $40(51.9 \%)$ & $37(48.1 \%)$ & & \\
\hline Age (year) & & & & 1.059 & 0.303 \\
\hline$<62$ & 58 & $33(56.9 \%)$ & $25(43.1 \%)$ & & \\
\hline$\geq 62$ & 47 & $22(46.8 \%)$ & $25(53.2 \%)$ & & \\
\hline Size $(\mathrm{cm})$ & & & & 0.159 & 0.690 \\
\hline$<5$ & 63 & $32(50.8 \%)$ & $31(49.2 \%)$ & & \\
\hline$\geq 5$ & 42 & $23(54.8 \%)$ & $19(45.2 \%)$ & & \\
\hline Location & & & & 0.153 & 0.927 \\
\hline Proximal & 33 & $18(54.5 \%)$ & $15(45.5 \%)$ & & \\
\hline Middle & 34 & $18(52.9 \%)$ & $16(47.1 \%)$ & & \\
\hline Distal & 38 & $19(50.0 \%)$ & $19(50.0 \%)$ & & \\
\hline Differentiation & & & & 0.095 & 0.757 \\
\hline Differentiated & 30 & $15(50.0 \%)$ & $15(50.0 \%)$ & & \\
\hline $\begin{array}{l}\text { Poorly } \\
\text { differentiated }\end{array}$ & 75 & $40(53.3 \%)$ & $35(46.7 \%)$ & & \\
\hline Invasion depth & & & & 0.239 & 0.625 \\
\hline $\mathrm{T} 1+\mathrm{T} 2$ & 21 & $12(57.1 \%)$ & $9(42.9 \%)$ & & \\
\hline $\mathrm{T} 3+\mathrm{T} 4$ & 84 & $43(51.2 \%)$ & $41(48.8 \%)$ & & \\
\hline $\begin{array}{l}\text { Lymph node } \\
\text { metastasis }\end{array}$ & & & & 0.095 & 0.757 \\
\hline No & 30 & $15(50.0 \%)$ & $15(50.0 \%)$ & & \\
\hline Yes & 75 & $40(53.3 \%)$ & $35(46.7 \%)$ & & \\
\hline $\begin{array}{l}\text { Distant } \\
\text { metastasis }\end{array}$ & & & & 5.674 & 0.017 \\
\hline No & 89 & $51(57.3 \%)$ & $38(42.7 \%)$ & & \\
\hline Yes & 16 & $4(25.0 \%)$ & $12(75.0 \%)$ & & \\
\hline TNM stage & & & & 0.600 & 0.439 \\
\hline $\mathrm{I}+\mathrm{II}$ & 38 & $18(47.4 \%)$ & $20(52.6 \%)$ & & \\
\hline III+IV & 67 & $37(55.2 \%)$ & $30(44.8 \%)$ & & \\
\hline
\end{tabular}




\subsection{Correlation between MACC1 expression and patients' prognosis}

Kaplan-Meier analysis showed that the survival rate of postoperative patients who tended to be MACC1-positive tended to be lower than that of the low MACC1 expression group. For all the patients, the 5-year survival rates were $22.0 \%(11 / 50)$ in the high MACC1 group while they were $52.7 \%(29 / 55)$ in the low MACC1 group. The median survival time was 40.14 and 27.84 months, respectively (Fig. 2).

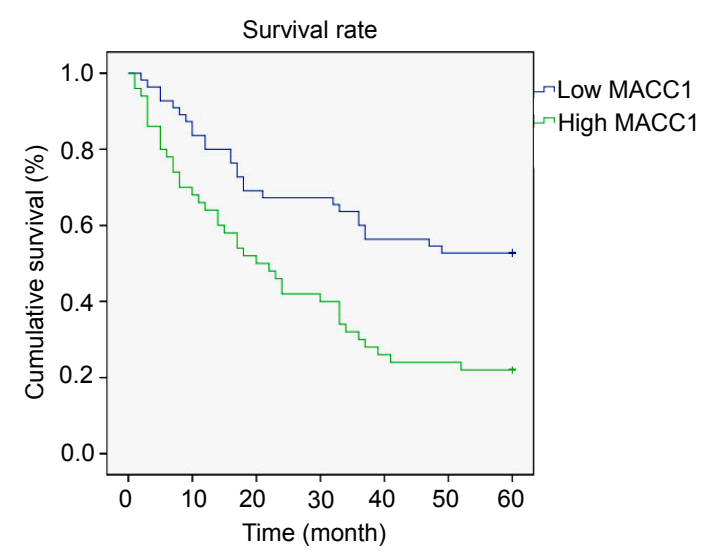

Fig. 2 Kaplan-Meier curves with univariate analyses for patients with low MACC1 expression versus high MACC1 expression tumors

\subsection{Diagram of MACC1-mediated signaling in GC}

MACC1 promotes cancer invasiveness and tumor growth during tumor development. Tumor development can be accelerated by the c-Met, Twist1/2, and VEGF pathways through MACC1 activation. Even worse than invasiveness, high MACC1 expression may lead to abnormal proliferation as a result of the promotion of tumor angiogenesis and the effect of cell-cycle deregulation. As expected, a higher recurrence and mortality rate are observed in GC patients with relatively positive MACC1 expression (Fig. 3).

\section{Discussion}

Some researchers suggest that MACC1 upregulation has a strong correlation with cancer development and progression in several types of solid tumors, including GC (Ge et al., 2011; Guo et al., 2013), hepatocellular carcinoma (Wang et al., 2013), lung adenocarcinoma (Wang et al., 2014), esophageal

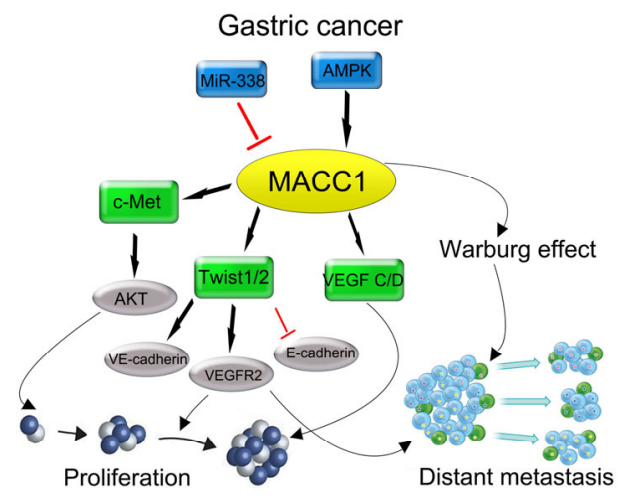

Fig. 3 Diagram summarizing the role of MACC1 in GC regulation during previous studies

cancer (Zhu et al., 2013), and breast cancer (Huang et al., 2013). However, the relation between MACC1 expression and patient survival across GC still remains controversial.

Until now, research via a group of GC patients and specimens had demonstrated that the MACC1 gene is closely related to cancer cell peritoneal dissemination and angiogenesis (Shirahata et al., 2010b; Wang et al., 2015). However, Ge et al. (2011) found that over-expression of MACC1 means better prognosis for GC patients. In this experiment, the relationship between patient prognosis and an oncological feature of this gene in GC was confirmed. Firstly, we demonstrated that the MACC1 protein was significantly highly expressed in GC. Furthermore, MACC1 upregulation was accompanied with short survival time and a higher possibility of cancer metastasis. Recently, various studies have concentrated on the studies of cancer around MACC1. In hepatocellular carcinoma, MACC1 is believed to be a prognostic factor for prognosis prediction (Qiu et al., 2011). Meanwhile, a high MACC1 presence was proved to have to be connected with recurrence after operation in lung adenocarcinoma (Shimokawa et al., 2011). Previous findings demonstrate the promotion effect of MACC1 in multiple cancer types, which support the clinical statistical results here. In conclusion, MACC1 ubiquitously promotes carcinogenesis, especially in gastrointestinal system source-derived cancers.

On the basis of our current experiments, we summarize the signaling regulation pathway of MACC1 during recent years (Fig. 3). MACC1 was initially identified as an activator of the HGF/c-Met 
pathway, which furthermore upregulated c-Met expression. As a result, CD44-HGF/c-Met and fibronectinHGF/c-Met may compose a feedback loop that positively enhances the GC cell EMT (Stein et al., 2009; Wang et al., 2013). Lin et al. (2015) reported that increased MACC1 expression correlates with metabolic stress in GC. To compensate, the existence of MACC1 ensures GC growth against metabolic stress by adjusting to the Warburg effect. Also, capillary vascular density was significantly increased in the tumors of patients who died of GC, and was positively correlated with MACC1 immunoreactivity analysis (Wang et al., 2015). Therefore, MACC1 may be applied as a new predictive molecular marker of GC, and down-regulation of MACC1 may provide a novel strategy for blocking the process of GC metastasis. Nevertheless, further experiments are needed to prove these hypotheses.

In conclusion, it was found that a high MACC1 presence correlated with the presence of GC metastasis and was closely related to patient prognosis. The listed factors suggest that MACC1 may serve as a parameter for the prognostic prediction factors of GC and is possibly a promising molecular target for GC treatment. Finally, the specific mechanisms involved in the progression of GC need further exploration.

\section{Compliance with ethics guidelines}

Qiu-ping XIE, Cheng XIANG, Gang WANG, Ke-feng LEI, and Yong WANG declare that they have no conflict of interest.

All procedures followed were in accordance with the ethical standards of the responsible committee on human experimentation (institutional and national) and with the Helsinki Declaration of 1975, as revised in 2008 (5). Informed consent was obtained from all patients for being included in the study. Additional informed consent was obtained from all patients for whom identifying information is included in this article.

\section{References}

Arlt, F., Stein, U., 2009. Colon cancer metastasis: MACC1 and Met as metastatic pacemakers. Int. J. Biochem. Cell Biol., 41(12):2356-2359. http://dx.doi.org/10.1016/j.biocel.2009.08.001

Chundong, G., Uramoto, H., Onitsuka, T., et al., 2011. Molecular diagnosis of MACC1 status in lung adenocarcinoma by IHC analysis. Anticancer Res., 31(4):1141-1145.

Edge, S.B., Compton, C.C., 2010. The American Joint Committee on cancer: the 7th edition of the AJCC cancer staging manual and the future of TNM. Ann. Surg. Oncol., 17(6):1471-1474.

http://dx.doi.org/10.1245/s10434-010-0985-4
Ge, S.H., Wu, X.J., Wang, X.H., et al., 2011. Over-expression of metastasis-associated in colon cancer-1 (MACC1) associates with better prognosis of gastric cancer patients. Chin. J. Cancer Res., 23(2):153-159. http://dx.doi.org/10.1007/s11670-011-0153-9

González, C.A., Sala, N., Capella, G., 2002. Genetic susceptibility and gastric cancer risk. Int. J. Cancer, 100(3):249-260. http://dx.doi.org/10.1002/ijc.10466

Guo, T., Yang, J., Yao, J., et al., 2013. Expression of MACC1 and c-Met in human gastric cancer and its clinical significance. Cancer Cell Int., 13(1):121. http://dx.doi.org/10.1186/1475-2867-13-121

Hamajima, N., Naito, M., Kondo, T., et al., 2006. Genetic factors involved in the development of Helicobacter pylori-related gastric cancer. Cancer Sci., 97(11):11291138. http://dx.doi.org/10.1111/j.1349-7006.2006.00290.x

Huang, N., Wu, Z., Lin, L., et al., 2015. MiR-338-3p inhibits epithelial-mesenchymal transition in gastric cancer cells by targeting ZEB2 and MACC1/Met/Akt signaling. Oncotarget, 6(17):15222-15234. http://dx.doi.org/10.18632/oncotarget.3835

Huang, Y., Zhang, H., Cai, J., et al., 2013. Overexpression of $\mathrm{MACC} 1$ and its significance in human breast cancer progression. Cell Biosci., 3(1):16-28. http://dx.doi.org/10.1186/2045-3701-3-16

Jemal, A., Bray, F., Center, M.M., et al., 2011. Global cancer statistics. CA Cancer J. Clin., 61(2):69-90. http://dx.doi.org/10.3322/caac.20107

Lee, J.H., Ryu, K.W., Kook, M.C., et al., 2008. Feasibility of laparoscopic sentinel basin dissection for limited resection in early gastric cancer. J. Surg. Oncol., 98(5): 331-335. http://dx.doi.org/10.1002/jso.21115

Lin, L., Huang, H., Liao, W., et al., 2015. MACC1 supports human gastric cancer growth under metabolic stress by enhancing the Warburg effect. Oncogene, 34(21):27002710 . http://dx.doi.org/10.1038/onc.2014.204

MacDonald, J.S., 2006. Gastric cancer-new therapeutic options. N. Engl. J. Med., 355(1):76-77. http://dx.doi.org/10.1056/NEJMe068121

Morabito, A., Carillio, G., Longo, R., 2009. Systemic treatment of gastric cancer. Crit. Rev. Oncol. Hematol., 70(3):216-234. http://dx.doi.org/10.1016/j.critrevonc.2008.08.005

Qiu, J., Huang, P., Liu, Q., et al., 2011. Identification of MACC1 as a novel prognostic marker in hepatocellular carcinoma. J. Transl. Med., 9(1):166. http://dx.doi.org/10.1186/1479-5876-9-166

Shimokawa, H., Uramoto, H., Onitsuka, T., et al., 2011. Overexpression of MACC1 mRNA in lung adenocarcinoma is associated with postoperative recurrence. J. Thorac. Cardiovasc. Surg., 141(4):895-898. http://dx.doi.org/10.1016/j.jtcvs.2010.09.044

Shirahata, A., Shinmura, K., Kitamura, Y., et al., 2010a. $\mathrm{MACC} 1$ as a marker for advanced colorectal carcinoma. 
Anticancer Res., 30(7):2689-2692.

Shirahata, A., Sakata, M., Kitamura, Y., et al., 2010b. MACC 1 as a marker for peritoneal-disseminated gastric carcinoma Anticancer Res., 30(9):3441-3444.

Shirahata, A., Fan, W., Sakuraba, K., et al., 2011. MACC 1 as a marker for vascular invasive hepatocellular carcinoma. Anticancer Res., 31(3):777-780.

Stein, U., Walther, W., Arlt, F., et al., 2009. MACC1, a newly identified key regulator of HGF-MET signaling, predicts colon cancer metastasis. Nat. Med., 15(1):59-67. http://dx.doi.org/10.1038/nm.1889

Sun, L., Duan, J., Jiang, Y., et al., 2015. Metastasis-associated in colon cancer-1 upregulates vascular endothelial growth factor-C/D to promote lymphangiogenesis in human gastric cancer. Cancer Lett., 357(1):242-253. http://dx.doi.org/10.1016/j.canlet.2014.11.035

Sun, P., Xiang, J.B., Chen, Z.Y., 2009. Meta-analysis of adjuvant chemotherapy after radical surgery for advanced gastric cancer. Br. J. Surg., 96(1):26-33. http://dx.doi.org/10.1002/bjs.6408

Wang, L., Wu, Y., Lin, L., et al., 2013. Metastasis-associated in colon cancer-1 upregulation predicts a poor prognosis of gastric cancer, and promotes tumor cell proliferation and invasion. Int. J. Cancer, 133(6):1419-1430. http://dx.doi.org/10.1002/ijc.28140

Wang, L., Lin, L., Chen, X., et al., 2015. Metastasis-associated in colon cancer-1 promotes vasculogenic mimicry in gastric cancer by upregulating TWIST1/2. Oncotarget, 6(13):11492-11506. http://dx.doi.org/10.18632/oncotarget.3416

Wang, Z., Li, Z., Wu, C., et al., 2014. MACC1 overexpression predicts a poor prognosis for non-small cell lung cancer. Med. Oncol., 31(1):790. http://dx.doi.org/10.1007/s12032-013-0790-6

Weidle, U.H., Birzele, F., Kruger, A., 2015. Molecular targets and pathways involved in liver metastasis of colorectal cancer. Clin. Exp. Metastasis, 32(6):623-635. http://dx.doi.org/10.1007/s10585-015-9732-3
Xu, S.T., Ding, X., Ni, Q.F., et al., 2015. Targeting MACC1 by RNA interference inhibits proliferation and invasion of bladder urothelial carcinoma in T24 cells. Int. J. Clin. Exp. Pathol., 8(7):7937-7944.

Zhou, X., Xu, C.J., Wang, J.X., et al., 2015. Metastasisassociated in colon cancer-1 associates with poor prognosis and promotes cell invasion and angiogenesis in human cervical cancer. Int. J. Gynecol. Cancer, 25(8): 1353-1363.

http://dx.doi.org/10.1097/IGC.0000000000000524

Zhu, M., Xu, Y., Mao, X., et al., 2013. Overexpression of metastasis-associated in colon cancer-1 associated with poor prognosis in patients with esophageal cancer. Pathol. Oncol. Res., 19(4):749-753. http://dx.doi.org/10.1007/s12253-013-9638-9

\section{中文概要}

题 目: $M A C C 1$ 高表达促进胃癌的转移, 缩短患者生存 时间

目 的: 评估 $M A C C 1$ 基因在胃癌发生发展中的作用。

创新点: 首次发现胃癌中 $M A C C 1$ 基因高表达, 并且在患 者标本中发现高表达 MACC1 和胃癌的远处转移 和预后有显著相关性。

方 法: 使用免疫组化的方法对 105 例胃癌患者进行分析, 并且将 MACC1 和其临床特点包括年龄、肿瘤大 小、肿瘤分化以及远处转移状况进行归纳和显著 性检验, 进一步将患者分为 MACC1 高表达和低 表达组。通过定期的随访, 绘制患者生存曲线。

结论: 本实验中发现了在胃癌远处转移灶组织中 MACC1 表达显著升高。然而 MACC1 的高表达 和胃癌的肿瘤大小、肿瘤分化等特性未见明显相 关性。同时胃癌患者的 5 年生存率和 MACC1 的 高表达有显著性关联。

关键词: 胃癌; $\mathrm{MACC} 1$; 预后; 远处转移 